\title{
One-Year Uptake of Pneumococcal Conjugate Vaccine: A National Survey of Family Physicians and Pediatricians
}

\author{
Matthew M. Davis, MD, MAPP, Serigne M. Ndiaye, PhD, Gary L. Freed, MD, MPH, \\ and Sarah J. Clark, MPH
}

Background: Pneumococcal conjugate vaccine (PCV7) was recommended by June 2000 for administration to all US children $\leq 23$ months old and to children at high risk for pneumococcal disease 24 to 59 months old. We sought to identify physician characteristics associated with adoption of the recommendation within 1 year.

Methods: We conducted a cross-sectional mail survey from April to July, 2001, of 788 family physicians (FP) and 833 pediatricians (PD) in 24 states. We measured whether physicians had adopted PCV7 recommendations, their expectations of the effectiveness of $\mathrm{PCV} 7$, the number of vaccine injections they would consider administering at 1 visit, and barriers to administering multiple injections.

Results: Response rate was $60 \%$. Overall, $87 \%$ of physicians had adopted PCV7 recommendations (68\% FP; 99\% PD; $P<.001)$. FP adopters are significantly more likely than nonadopters to believe PCV7 will be effective in preventing pneumococcal sepsis and meningitis, as well as $\mathbf{2 5 \%}$ of cases of otitis media. In multivariable logistic regression analyses of adoption of PCV7, FP who have higher proportions of African American patients and patients on Medicaid, see greater numbers of newborns, work in practices of $\geq 4$ physicians, and are willing to consider administering at least 4 vaccine injections at 1 visit are significantly more likely to have adopted PCV7. Concerns about vaccine cost and reimbursement were the most commonly cited factors in physicians' decisions not to adopt PCV7 recommendations.

Conclusions: One year after PCV7 was recommended, nearly all pediatricians and a majority of family physicians had incorporated this vaccine into their practices. Barriers to higher rates of uptake-especially among family physicians - must be addressed to achieve immunization goals with this new vaccine. (J Am Board Fam Pract 2003;16:363-71.)

In the year 2000, 7-valent pneumococcal conjugate vaccine (PCV7; pneumococcal-CRM197; Prevnar, Wyeth-Ayerst, Princeton, NJ) was recommended for administration to all children under 2 years of age and to high-risk children from 2 to 5 years of

Submitted, revised, 18 November 2000.

From the Child Health Evaluation and Research (CHEAR) Unit, Division of General Pediatrics (MMD, GLF, SJC), and Division of General Internal Medicine (MMD), University of Michigan, Ann Arbor; and National Immunization Program, Centers for Disease Control and Prevention, Atlanta, Georgia (SMN). Address correspondence to Matthew M. Davis, MD, MAPP, University of Michigan, 300 NIB, 6C23, Ann Arbor, MI 48109-0456 (e-mail: mattdav@umich.edu).

This work was funded by the Centers for Disease Control and Prevention through a cooperative agreement with the Association of Teachers of Preventive Medicine. This work was presented in part at the annual meeting of the Pediatric Academic Societies, Baltimore, MD, May 2002. age by the American Academy of Family Physicians (AAFP),${ }^{1}$ the American Academy of Pediatrics $(\mathrm{AAP}),{ }^{2}$ and the Advisory Committee on Immunization Practices (ACIP) of the Centers for Disease Control and Prevention. ${ }^{3}$ The 4-dose series of PCV7 has been demonstrated to be safe ${ }^{4}$ and effective in preventing more than $97 \%$ of invasive pneumococcal disease in children. ${ }^{5}$ In addition, randomized controlled trials have indicated that PCV7 vaccination significantly reduces the frequency of acute otitis media by $7 \%$ overall $^{5}$ and by $34 \%$ in culture-verified pneumococcal cases. ${ }^{6}$

The clinical promise of PCV7 may be tempered by its purchase price and the overall number of vaccines now recommended for young children. The addition of PCV7 to the universal childhood immunization schedule nearly doubled the overall cost of all recommended vaccines up to age 6 
years. ${ }^{7}$ The initial average wholesale price of $\$ 58$ per dose led some physicians to require that families sign affidavits of financial responsibility that held them accountable if private insurers did not reimburse adequately. ${ }^{8}$

Another concern is that the addition of $\mathrm{PCV} 7$ to the harmonized schedule means that as many as 5 separate injections may need to be administered at the 2- and 4-month routine health supervision visits. ${ }^{9}$ Recent studies suggest that many physicians and parents will accept as many as 4 injections at 1 visit, ${ }^{10}$ but comments from physicians in the months immediately after $\mathrm{PCV} 7$ recommendations in $2000^{8}$ underscored growing concerns about the "pincushion effect" that may be a barrier to broad acceptance of newly recommended vaccines. ${ }^{11,12}$

These conflicting factors have raised concerns about adoption of PCV7 by physicians. Prior studies indicate that adoption of new childhood vaccine recommendations is associated with physician factors such as specialty, proportion of Medicaid patients, practice ownership, beliefs that parents or nurses will object to the new vaccine, and adherence to national vaccine recommendations. ${ }^{13-21}$ We conducted a national survey of family physicians and pediatricians within 1 year after the initial recommendations for PCV7 were issued to investigate physician factors associated with adoption of PCV7.

\section{Methods \\ Design}

The study was a multistate, cross-sectional mail survey of pediatricians and family physicians who provide primary care to children $\leq 5$ years of age. The study protocol was approved by the Institutional Review Boards of the University of Michigan Medical School and the Centers for Disease Control and Prevention (CDC).

\section{Sample}

Random samples of 788 family physicians and 833 pediatricians were drawn from the American Medical Association (AMA) Physician Masterfile by a contracted vendor (Medical Marketing Services). The Masterfile is the most comprehensive listing of physicians in the United States and contains both members and nonmembers of the AMA.

Sampling was conducted in 24 states, selected for their geographic diversity and vaccine financing strategy regarding PCV7, obtained from the National Immunization Program of CDC. States that have a "Vaccines for Children (VFC)-only" strategy (AR, CA, IN, MS, NJ, NV, PA, UT, VA, VT, WI, WV) purchase vaccine only for children who qualify for VFC (Medicaid beneficiaries, the uninsured, Native Alaskans and Native Americans, and underinsured children receiving vaccine at rural health clinics or federally qualified health centers). In states with "enhanced VFC" strategy (GA, MD, MI, MN, NY, OK, TX), the state purchases vaccine for VFC-eligible children as well as for underinsured children seen in private practice. In universal purchase states (ME, NC, NH, NM, WA), the state purchases vaccine for all children, regardless of insurance status. Sampling was conducted from each state proportional to physician representation in the Masterfile. Excluded from the sampling frame were physicians $\geq 70$ years old and resident physicians.

\section{Survey Instrument}

Two separate survey instruments were designed: 1 for physicians who had adopted PCV7 recommendations for children $<2$ years old and 1 for physicians who had not. Several questions were common to both, allowing direct comparison of multiple physician characteristics and attitudes between adopters and nonadopters. Physicians' specialty, gender, year of graduation from medical school, and board certification status were obtained from the AMA Masterfile.

\section{Survey Administration}

Before mailing, survey items were pilot-tested with family physicians and pediatricians. The survey was initially mailed with a personalized cover letter and a \$3 incentive in April 2001. Nonrespondents were contacted with additional letters and surveys in May and June, 2001.

\section{Data Analysis}

All responses received by the study closure date of July 15, 2001, were included in the analysis. All analyses were conducted using SAS (version 6.12; SAS Institute, Cary, NC). Tests of significance in bivariate comparisons were computed using likelihood ratio $\chi^{2}$, except in cases of zero values in 1 or more cells, where Fisher's exact test was used. Variables associated with adopting PCV7 in bivariate analyses were entered into 2 separate multivariate 


\begin{tabular}{|c|c|c|c|}
\hline Practice Characteristic & $\begin{array}{l}\text { Pediatricians } \\
(\mathrm{n}=435)(\%)\end{array}$ & $\begin{array}{l}\text { Family Physicians } \\
(\mathrm{n}=259)(\%)\end{array}$ & $P$ Value \\
\hline \multicolumn{4}{|l|}{ Ownership of practice } \\
\hline University & 4 & 3 & \multirow{6}{*}{$>.8$} \\
\hline Hospital & 12 & 15 & \\
\hline Physician network* & 11 & 10 & \\
\hline Public & 3 & 4 & \\
\hline Independent private & 69 & 68 & \\
\hline Other & 1 & 0 & \\
\hline \multicolumn{4}{|c|}{ Number of physicians in practice } \\
\hline 1 & 17 & 22 & \multirow[t]{5}{*}{$<.01$} \\
\hline $2-3$ & 23 & 32 & \\
\hline $4-5$ & 23 & 15 & \\
\hline $6-9$ & 18 & 17 & \\
\hline$\geq 10$ & 19 & 14 & \\
\hline \multicolumn{4}{|c|}{ Proportion of patients on Medicaid } \\
\hline$<10 \%$ & 37 & 46 & \multirow[t]{4}{*}{$<.05$} \\
\hline $10 \%-40 \%$ & 36 & 36 & \\
\hline $41 \%-70 \%$ & 17 & 12 & \\
\hline$>70 \%$ & 10 & 6 & \\
\hline \multicolumn{4}{|c|}{ Proportion of patients who are African American } \\
\hline$<10 \%$ & 55 & 69 & \multirow[t]{3}{*}{$=.001$} \\
\hline $10 \%-40 \%$ & 38 & 25 & \\
\hline$>40 \%$ & 7 & 6 & \\
\hline \multicolumn{4}{|c|}{ Number of newborns enrolled in practice annually } \\
\hline$<20$ & 2 & 49 & \multirow{3}{*}{$<.001$} \\
\hline $20-40$ & 18 & 33 & \\
\hline$>40$ & 80 & 18 & \\
\hline \multicolumn{4}{|c|}{ Vaccine financing strategy of state in which physician practices $\dagger$} \\
\hline VFC only & 46 & 43 & \multirow{3}{*}{$>.4$} \\
\hline Enhanced VFC & 44 & 45 & \\
\hline Universal purchase & 10 & 12 & \\
\hline
\end{tabular}

Within each specialty for each characteristic, column percentages sum to 100 .

* Includes physicians reporting practice ownership by health maintenance organization.

† Specifically regarding PCV7.

logistic regression models, based on the work of Cabana et $\mathrm{al}^{22}$ regarding barriers to physician adoption of practice guidelines. Model 1 compared the relative importance of variables characterizing physicians' practice characteristics for adoption of PCV7. Model 2 compared variables representing physicians' prior clinical experiences and attitudes that might influence their adoption of PCV7.

\section{Results}

\section{Characteristics of Respondents}

Among 1621 physicians in the initial sample, 458 of 788 family physicians (FP) (58\%) and 507 of 833 pediatricians (PD) $(61 \%)$ responded, for an overall response rate of $60 \%$. There were no significant differences between respondents and nonrespondents in gender, age, year of graduation from medical school, board certification, or state in which they practice. Some respondents were ineligible for analysis because they did not see children under age 5 years (157 FP, $53 \mathrm{PD})$, had retired (16 FP, $5 \mathrm{PD})$, or for other miscellaneous reasons, such as practice in the uniformed services or a suspended or revoked license (26 FP, $14 \mathrm{PD})$.

Our sample therefore contained $259 \mathrm{FP}$ and 435 PD eligible for analysis. Of these physicians, $40 \%$ were women and $85 \%$ were board-certified in their clinical specialties. Although $26 \%$ graduated from medical school within the past 10 years, $11 \%$ graduated more than 30 years ago. Approximately $45 \%$ practice in states with a VFC-only strategy for PCV7 purchase, $44 \%$ in states with enhanced VFC financing, and $11 \%$ in states with universal purchase regarding PCV7. FP and PD respondents differed in several practice characteristics (Table 1).

\section{Adoption of PCV7 Recommendations}

At the time of the survey, $87 \%$ of respondents had adopted PCV7 recommendations for children $<2$ years old (68\% FP and 99\% PD; $P<.001)$. The most common schedule used for administration of PCV7 was visits at 2, 4, 6, and $>12$ months of life 


\begin{tabular}{|c|c|c|c|}
\hline \multirow[b]{2}{*}{ For Children Aged 24-59 Months Old Who } & \multicolumn{3}{|c|}{$\begin{array}{c}\text { Percentage of Physicians Who Have Adopted } \\
\text { PCV7 in Practice }\end{array}$} \\
\hline & $\begin{array}{c}\text { Always } \\
\text { Recommend } \\
\text { PCV7 }\end{array}$ & $\begin{array}{l}\text { Sometimes } \\
\text { Recommend } \\
\text { PCV7 }\end{array}$ & $\begin{array}{c}\text { Rarely/Never } \\
\text { Recommend } \\
\text { PCV7 }\end{array}$ \\
\hline Have chronic illnesses that affect immunity to infection* & 93 & 5 & 2 \\
\hline Have recurrent otitis media* & 51 & 34 & 15 \\
\hline Attend daycare $†$ & 44 & 37 & 19 \\
\hline Are African American & 39 & 36 & 26 \\
\hline Are NOT identified as high-risk & 27 & 35 & 38 \\
\hline
\end{tabular}

* Physician behavior for this high-risk category significantly different from physician behavior for standard-risk 24- to 59-month-olds (bottom row) at $p<.001$.

† Physician behavior for this high-risk category significantly different from physician behavior for standard-risk 24- to 59-month-olds at $p<.01$.

(61\%). Administration at 2, 4, 6, and 12 months of life was less common (34\%), and a few physicians used a combination of visits at other months to administer PCV7 (5\%).

Approximately $95 \%$ of physicians who administer PCV7 at the 2-, 4-, and 6-month visits reported that they administered $\geq 4$ vaccine injections at the 2 -month visit. In contrast, $93 \%$ of physicians who administer vaccines during extra visits gave $\leq 3$ injections at the 2 -month visit.

More than half $(58 \%)$ of physicians who had adopted PCV7 said they had made no changes in their typical immunization schedule as a result of adding the vaccine. In contrast, $22 \%$ said that they now more often give combination hepatitis B/ Haemophilus influenzae, type b vaccine, $14 \%$ said that they administer hepatitis B vaccine at birth and/or 1-month visits more frequently, and 12\% reported that they now postpone either measles/ mumps/rubella or the varicella vaccine until 15 months or later to give PCV7.

For older children aged 24 to 59 months, physicians who had adopted PCV7 differed in their approaches to recommending PCV7 for groups of children at increased risk for pneumococcal disease (Table 2). Almost all who administered PCV7 recommended the vaccine for immuno-compromised children, but there was substantial variation in patterns of physician recommendation regarding other high-risk categories. Physicians were significantly more likely to recommend PCV7 for children with recurrent otitis media and those in day care than for children of the same age who are not in a designated risk category. In contrast, overall physician recommendations for African American children were not significantly different from recommendations for standard-risk children.

\section{Family Physician Factors Associated with Adoption of PCV7}

Nearly all PD in this sample had adopted PCV7, so physician factors that distinguish PCV7 adopters (ADOP) from nonadopters (N-ADOP) are described only for $\mathrm{FP}$ respondents.

Practice characteristics of FP ADOP appear in Table 3. In general, FP who were in larger practices, cared for more newborns annually, and had practices with higher proportions of African American children and Medicaid beneficiaries were more likely to have adopted PCV7. In addition, female $\mathrm{FP}$ are more likely to have adopted PCV7 than male FP (78\% vs $64 \% ; P<.05)$. No differences were apparent among $\mathrm{FP}$ working in states with different vaccine financing strategies for PCV7 or in practices with different types of ownership or affiliation.

Attitudes and prior clinical experiences associated with physicians' adoption of PCV7 fell into 3 categories: comfort with multiple injections at 1 visit, anticipated effectiveness of $\mathrm{PCV} 7$, and the influence of experiences with rotavirus vaccine. $\mathrm{FP}$ ADOP were more likely than $\mathrm{N}-\mathrm{ADOP}$ to consider giving 4 or more vaccine injections at 1 visit $(86 \%$ vs $35 \% ; P<.001) ; 49 \%$ of $\mathrm{N}-\mathrm{ADOP}$ said they would consider giving only 3 injections at a visit, and $16 \%$ administer 2 or fewer. ADOP were less likely to be concerned that multiple injections could cause adverse effects ( $26 \%$ vs $43 \% ; P<.01)$. 


\begin{tabular}{lc}
\hline Practice Characteristics & Have Adopted PCV7 $(n=176)(\%)$ \\
\hline Number of physicians at practice site & \\
1 & 54 \\
2 & 64 \\
3 & 55 \\
4 & 63 \\
$5-10$ & 82 \\
$>10$ & 81 \\
Proportion of patients $<5$ years old covered by Medicaid & \\
$<10 \%$ & 57 \\
$10 \%-40 \%$ & 73 \\
$>40 \%$ & 85 \\
Proportion of patients who are African-American & \\
$<10 \%$ & 64 \\
$10 \%-40 \%$ & 75 \\
$>40 \%$ & 93 \\
Annual newborn enrollment & \\
$<20$ & 57 \\
$20-40$ & 71 \\
$>40$ & 91 \\
\hline
\end{tabular}

$P$ values indicate level of significance for comparison of proportions of adopters among physicians with different characteristics within each category.

ADOP were also less likely to express their own concerns about too much pain with multiple injections $(49 \%$ vs $78 \% ; P<.001)$, and less likely to report parents' concerns about multiple injections ( $65 \%$ vs $80 \% ; P<.05)$. ADOP and $\mathrm{N}-\mathrm{ADOP}$ were equally likely (49\%) to be aware of nurses' concerns about multiple injections.

Family physicians' expectations about the effectiveness of PCV7 in preventing pneumococcal disease also distinguish ADOP from N-ADOP (Table 4). ADOP were significantly more likely to expect PCV7 to be effective against pneumococcal meningitis, pneumococcal sepsis, and otitis media. ADOP were also more likely than N-ADOP to have experienced a patient death or severe morbidity attributable to pneumococcal disease $(30 \%$ vs $21 \%$ ), although this finding only reached borderline statistical significance.
ADOP were significantly less likely to say that their experiences with rotavirus vaccine had influenced their approach to PCV7 (33\% vs $46 \% ; P<$ $.05)$. Among physicians for whom rotavirus vaccine experiences had been influential, ADOP were more likely to say that they spend more time discussing vaccine risks with parents as a result (54\% vs $19 \%$; $P<.001)$.

In separate multivariable logistic regression models, we compared the relative importance of several factors associated with the adoption of PCV7 by FP. The results of the models are shown in Table 5. Among practice characteristics, the number of newborns seen annually was the most influential factor predisposing to PCV7 adoption. Among physician attitudes and clinical experiences, the number of injections a physician would consider giving at a single visit was of greatest importance.

Table 4. Differences between Family Physician Adopters and Nonadopters in Expectations of PCV7 Effectiveness in Preventing Disease

\begin{tabular}{|c|c|c|c|c|}
\hline & \multicolumn{4}{|c|}{ Percentage Who Believe PCV7 Will Effectively Prevent } \\
\hline & $\begin{array}{c}\text { Pneumococcal } \\
\text { Meningitis* }\end{array}$ & $\begin{array}{c}\text { Pneumococcal } \\
\text { Sepsis }^{*}\end{array}$ & $\begin{array}{c}\text { Pneumococcal } \\
\text { Pneumonia }\end{array}$ & $\begin{array}{l}>25 \% \text { of All } \\
\text { Otitis Media } †\end{array}$ \\
\hline Adopters of PCV7 & 47 & 43 & 33 & 33 \\
\hline Nonadopters of PCV7 & 25 & 23 & 25 & 22 \\
\hline
\end{tabular}

* Comparison of adopters versus non-adopters significant at $P<.001$.

† Comparison of adopters versus non-adopters significant at $P<.05$. 
Table 5. Multivariable Logistic Regression of Family Physician Adoption of PCV7 Recommendations

\begin{tabular}{lcc}
\hline Model/Variables & Odds Ratio & $95 \%$ Confidence Interval \\
\hline Model $1-$ Practice characteristics* & & $(1.20,11.01)$ \\
Number of newborns annually $(>40) \dagger$ & 3.64 & $(1.27,4.04)$ \\
Prop. patients on Medicaid $(\geq 0 \%) \dagger$ & 2.26 & $(1.21,3.98)$ \\
Number of physicians $(\geq 4) \dagger$ & 2.19 & $(1.10,4.15)$ \\
Prop. patients African American $(\geq 10 \%) \dagger$ & 2.13 & $(0.82,3.22)$ \\
Female physician & 1.62 & $(6.35,47.05)$ \\
Model 2-Clinical experiences \& attitudes $\dagger$ & & $(4.21,17.94)$ \\
Considers giving $\geq 5$ injections at 1 visit $\dagger$ & 17.29 & $(0.93,3.73)$ \\
Considers giving 4 injections at 1 visit $\dagger$ & 8.69 & $(0.66,3.17)$ \\
Thinks PCV7 will effectively prevent pneumococcal meningitis & 1.86 & $(0.55,2.17)$ \\
Has experienced severe patient morbidity or mortality from & 1.45 & $(0.22,0.86)$ \\
pneumococcal disease & & 1.09 \\
PCV7 approach influenced by rotavirus vaccine & 0.43 & \\
Reports $\geq 3$ problems with multiple injections $\dagger$ & & \\
\hline
\end{tabular}

* Reference group is male family physician with $<4$ physicians in practice, $<10 \%$ of patients on Medicaid, $<10 \%$ African American patients, who enrolls $\leq 40$ newborns annually in her/his panel.

† Statistically significant association with PCV7 adoption.

$\ddagger$ Reference group is family physician who will consider giving $<4$ vaccine injections at 1 visit, does not think PCV7 will be effective in preventing pneumococcal meningitis, has not experienced patient morbidity and/or mortality from pneumococcal disease, was not influenced by rotavirus vaccine, and reports $<3$ problems with multiple injections.

\section{Deterrents to Physician Adoption of PCV7 Recommendations}

Family physicians who had not adopted PCV7 at the time of this survey provided information about factors that were important in their decisions not to recommend this vaccine. Lack of insurance reimbursement for PCV7 was the most commonly cited factor (78\%). Sixty-four percent of N-ADOP said that the vaccine purchase costs were too much for their practices to bear, and $62 \%$ said that they were concerned about possible adverse events related to the vaccine. Over half (56\%) stated that they were unconvinced that a vaccine is necessary to combat pneumococcal disease; ie, they believed that antibiotics are sufficient to battle pneumococcal illness once it develops.

\section{Discussion}

The major finding of this nationwide survey is that, within 1 year of the announcement of harmonized recommendations from AAFP, AAP, and ACIP, $87 \%$ of child health care providers- $68 \%$ of family physicians and $99 \%$ of pediatricians- had adopted PCV7 recommendations for their patients under 2 years of age. Similarly rapid rates of adoption of a newly recommended vaccine into practice have been documented previously, but not on a national scale. For H. influenzae, type b, vaccine in 1986, uptake by pediatricians within 6 to 8 months was $86 \%$ and by family physicians was $61 \%$ in a New Mexico sample. ${ }^{23}$ Two-year uptake of universal hepatitis B recommendations was $90 \%$ among pediatricians and $64 \%$ among family physicians in 9 states by $1993 .{ }^{15}$ In contrast, pediatrician incorporation of varicella vaccine into practice within 1 to 2 years in a Washington state sample was only $44 \%{ }^{24}$

Although childhood vaccination rates are affected by multiple factors including family and health system issues as well as physician behaviors, ${ }^{25,26}$ physician adoption of national recommendations is essential to the success of immunization initiatives. Recent data indicate a clear link between early physician incorporation of vaccines into routine practice and subsequent achievement of national immunization goals. Hepatitis B recommendations issued in 1991 were adopted at rates similar to those found for PCV7 in this study; 5 years later, $82 \%$ of children aged 19 to 35 months in the US had received their full 3-dose hepatitis B series. In contrast, lower physician adoption rates of varicella vaccine contributed to a lower immunization rate $(67 \%)$ for this vaccine 5 years after it was recommended. ${ }^{27}$ When uptake rates are high and immunization goals are met quickly after a new vaccine is recommended for universal administration to chil- 
dren, children and communities realize more rapidly the reductions in morbidity and mortality associated with use of the vaccine. A recent example of rapid uptake leading to pronounced reductions in preventable disease is the Hib vaccine. ${ }^{28}$

Prior studies have reported that family physicians as a group adopt new childhood vaccine recommendations more slowly than pediatricians. ${ }^{14,15,18-21}$ We found the same pattern in this national sample regarding PCV7. Our findings also illustrate that attitudes regarding the administration of multiple vaccine injections at a single office visit distinguish PCV7 adopters from nonadopters, similar to findings reported by Kimmel et $\mathrm{al}^{21}$ among family physicians regarding polio vaccine. Physicians who perceive fewer barriers to multiple injections-eg, parental concerns, fears of adverse events, or their own concerns about pain from so many injections-are more likely to have adopted PCV7. A small minority of physicians who are not comfortable with 4 or 5 simultaneous injections evidently feel strongly enough about the benefits of PCV7 to schedule additional office visits. Additional visits will require more efforts from parents and physicians to be successful; the degree to which this strategy may increase health care costs is not yet clear.

Although physicians remain concerned about the degree to which infants today are "pincushions" from today's immunization schedule, our findings suggest there has been an evolution in physicians' sense of how many injections parents will tolerate. When universal hepatitis B immunization was recommended in 1991, about one-quarter of physicians believed that the majority of parents would object to 3 injections at 1 time. ${ }^{13}$ By the time Woodin et $\mathrm{al}^{11}$ characterized "pincushion" concerns in 1995, most physicians expressed concerns about administering 4 injections at once. In our study, $95 \%$ of physicians who have adopted PCV7 administer 4 or more vaccines at the 2 -month visit.

Physician uptake of new vaccine recommendations has been conceptualized as a process that includes 4 steps: awareness of recommendations, agreement with recommendations, the decision to adopt the recommendations, and finally adherence to recommendations. ${ }^{16}$ Our prior work regarding PCV7 among physicians in 7 states suggested that pediatricians and family physicians were aware of vaccine recommendations immediately after they were announced in late spring $2000 .^{8}$ Data in the study reported here indicate that some physicians are not recommending PCV7 because they do not agree that vaccination is necessary to address pneumococcal disease. However, $87 \%$ of physicians overall have agreed with the PCV7 guidelines, adopted them, and are adhering to the guidelines for children $\leq 23$ months of age. Our multivariable model indicates that physician perceptions of the clinical effectiveness of PCV7 are less important than their comfort with multiple injections. Even so, it is remarkable that one-third of family physicians who have adopted PCV7 in their practices believe that the vaccine will prevent more than $25 \%$ of otitis media cases-much greater than the $7 \%$ of cases prevented in clinical trials. ${ }^{5}$

Physician adherence to harmonized PCV7 recommendations for older children ${ }^{3}$ is less consistent, which may reflect differing levels of agreement among physicians about the characterization of high-risk groups, such as children in day care and African Americans. Higher rates of uptake among family physicians who see higher proportions of African American patients and Medicaid beneficiaries may reflect the positive effects of the VFC program that provides government-purchased PCV7 for all children on Medicaid, about $35 \%$ of whom are African American. ${ }^{29}$

Physicians who have not adopted PCV7 in their practices expressed concerns about the cost of PCV7 and lack of reimbursement from insurers. Given these concerns, it is somewhat surprising that the state vaccine financing strategy regarding PCV7 was not associated with physician recommendation of this vaccine. States' vaccine financing strategies may not influence overall physician adoption of PCV7 but may affect whether physicians administer the vaccine to children without insurance coverage for the vaccine in their practices.

Limitations to this study include its scope and its timing. We may have over- or underestimated the PCV7 uptake rate if responders differ from nonresponders in their adoption of PCV7. Our response rate was similar to those of other national mail surveys of physicians. ${ }^{30,31}$ In addition, this survey was completed before shortages of PCV7 vaccine became apparent on a national level and ACIP recommendations were changed to prioritize doses among the youngest and highest-risk children eligible. ${ }^{32}$ How the shortage of PCV7 will ultimately affect rates of uptake is not yet clear. 
In conclusion, our analysis offers insight into how PCV7 uptake might be improved further. Future efforts must focus on barriers perceived predominantly by family physicians, addressing their objections to multiple vaccine injections and their concerns about vaccine cost and reimbursement. Uptake rates of newly recommended childhood vaccines in the future may be enhanced if studies of physicians' perceived barriers-both in general and specific to the new vaccine in question-are conducted before consensus recommendations are issued, rather than after the fact.

Nearly half of all physicians who provide routine health care to children in the US are family physicians, ${ }^{33}$ and their acceptance and adoption of immunization recommendations are essential to protect the health of the nation's children. The barriers that family physicians perceive may not be unique to PCV7, particularly as we anticipate the recommendation of childhood vaccines in the future that may be more expensive than PCV7. ${ }^{34}$ In fact, experiences with PCV7 uptake may be indicative of barriers that will become even more salient as more vaccines are added to the childhood immunization schedule in the future.

\section{References}

1. American Academy of Family Physicians. AFP clinical recommendations: pneumococcal conjugate immunization. Available at: URL: http://www.aafp.org/ x1556.xml.

2. Anonymous. American Academy of Pediatrics. Committee on Infectious Diseases. Policy statement: recommendations for the prevention of pneumococcal infections, including the use of pneumococcal conjugate vaccine (Prevnar), pneumococcal polysaccharide vaccine, and antibiotic prophylaxis. Pediatrics 2000;106:362-6.

3. Advisory Committee on Immunization Practices. Preventing pneumococcal disease among infants and young children. Recommendations of the Advisory Committee on Immunization Practices (ACIP). MMWR Recomm Rep 2000;49(RR-9):1-35.

4. Rennels MB, Edwards KM, Keyserling HL, et al. Safety and immunogenicity of heptavalent pneumococcal vaccine conjugated to CRM197 in United States infants. Pediatrics 1998;101:604-11.

5. Black S, Shinefield HR, Fireman B, et al. Efficacy, safety and immunogenicity of heptavalent pneumococcal conjugate vaccine in children. Pediatr Infect Dis J 2000;19:187-95.

6. Eskola J, Kilpi T, Palmu A, et al. Efficacy of a pneumococcal conjugate vaccine against acute otitis media. N Engl J Med 2001;344:403-9.
7. Centers for Disease Control. Vaccine price list. Available at: URL: http://www.cdc.gov/nip/vfc/ cdc_vac_price_list.htm.

8. Davis MM, Andreae M, Freed GL. Physicians' early challenges related to the pneumococcal conjugate vaccine. Ambul Pediatr 2001;1:302-5.

9. Anonymous. Recommended childhood immunization schedule-United States, 2001. MMWR Morb Mortal Wkly Rep 2001;50:7-10, 19.

10. Lieu TA, Davis RL, Capra AM, et al. Variation in clinician recommendations for multiple injections during adoption of inactivated polio vaccine. Pediatrics 2001;107:e49.

11. Woodin KA, Rodewald LE, Humiston SG, Carges MS, Schaffer SJ, Szilagyi PG. Physician and parent opinions: are children becoming pincushions for immunizations? Arch Pediatr Adolesc Med 1995;149: 845-9.

12. Kolasa MS, Petersen TJ, Brink EW, Bulim ID, Stevenson JM, Rodewald LE. Impact of multiple injections on immunization rates among vulnerable children. Am J Prev Med 2001;21:261-6.

13. Freed GL, Bordley WC, Clark SJ, Konrad TR. Reactions of pediatricians to a new Centers for Disease Control recommendation for universal immunization of infants with hepatitis B vaccine. Pediatrics 1993;91:699-702.

14. Freed GL, Bordley WC, Clark SJ, Konrad TR. Universal hepatitis $\mathrm{B}$ immunization of infants: reactions of pediatricians and family physicians over time. Pediatrics 1994;93:747-51.

15. Freed GL, Freeman VA, Clark SJ, Konrad TR, Pathman DE. Pediatrician and family physician agreement with and adoption of universal hepatitis B immunization. J Fam Pract 1996;42:587-92.

16. Pathman DE, Konrad TR, Freed GL, Freeman VA, Koch GG. The awareness-to-adherence model of the steps to clinical guideline compliance. Med Care 1996;34:873-89.

17. Freed GL, Clark SJ, Konrad TR, Pathman DE. Factors affecting physicians' early adoption of combined DTP-Hib vaccine. Ambulatory Child Health 1997;3:27-33.

18. Ipp M, Macarthur C, Winders P, Gold R. Influenza vaccination of high-risk children: a survey of three physician groups. Can J Public Health 1998;89: 415-8.

19. Takayama JI, Iser JP, Gandelman A. Regional differences in infant immunization against hepatitis B: did intervention work? Prev Med 1999;28:160-6.

20. Schaffer SJ, Bruno S. Varicella immunization practices and the factors that influence them. Arch Pediatr Adolesc Med 1999;153:357-62.

21. Kimmel S, Puczynski S, McCoy RC, Puczynski MS. Practices of family physicians and pediatricians in administering poliovirus vaccine. J Fam Pract 1999; 48:594-600.

22. Cabana MD, Rand CS, Powe NR, et al. Why don't 
physicians follow clinical practice guidelines? A framework for improvement. JAMA 1999;282:145865.

23. Cochi SL, Fleming DW, Hull HF, Preblud SR, Orenstein WA. Haemophilus influenzae b polysaccharide vaccine. Physician acceptance and use of a new vaccine. Am J Dis Child 1986;140:1226-30.

24. Newman RD, Taylor JA. Reactions of pediatricians to the recommendation for universal varicella vaccination. Arch Pediatr Adolesc Med 1998;152:792-6.

25. Shefer A, Briss P, Rodewald L, et al. Improving immunization coverage rates: an evidence-based review of the literature. Epidemiol Rev 1999;21:96142.

26. Briss PA, Rodewald LR, Hinman AR, et al. Reviews of evidence regarding interventions to improve vaccination coverage in children, adolescents, and adults. The Task Force on Community Preventive Services. Am J Prev Med 2000;18(1 Suppl):97-140.

27. Anonymous. National, state, and urban area vaccination coverage levels among children aged 19-35 months-United States, 2000. MMWR Morb Mortal Wkly Rep 2001;50:637-41.

28. Zhou F, Bisgard KM, Yusuf HR, Deuson RR, Bath SK, Murphy TV. Impact of universal Haemophilus influenzae type b vaccination starting at 2 months of age in the United States: an economic analysis. Pediatrics 2002;110:653-61.

29. U.S. Census Bureau. Health insurance detailed table: 2000. Current Population Survey. Available at: http://www.census.gov/hhes/hlthins/hlthin00/ dtable10.html.

30. Asch DA, Jedrziewski K, Christakis NA. Response rates to mailed surveys published in medical journals. J Clin Epidemiol 1997;50:1129-36.

31. Cummings SM, Savitz LA, Konrad TR. Reported response rates to mailed physician questionnaires. Health Serv Res 2001;35:1347-55.

32. Anonymous. From the Centers for Disease Control and Prevention. Updated recommendations on the use of pneumococcal conjugate vaccine in a setting of vaccine shortage-Advisory Committee on Immunization Practices. JAMA 2002;287:833-4.

33. LeBaron CW, Massoudi M, Stevenson J, Lyons B. Vaccination coverage and physician distribution in the United States, 1997. Pediatrics 2001;107:e31.

34. Davis MM, Zimmerman JL, Wheeler JR, Freed GL. Childhood vaccine purchase costs in the public sector: past trends, future expectations. Am J Public Health 2002;92:1982-7. 